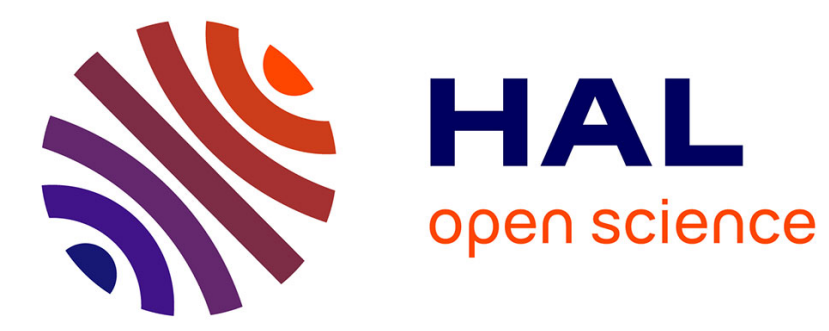

\title{
How Do Typically Developing Children and Children with ASD Play a Tangible Game?
}

\author{
Amani Indunil Soysa, Abdullah Al Mahmud
}

\section{To cite this version:}

Amani Indunil Soysa, Abdullah Al Mahmud. How Do Typically Developing Children and Children with ASD Play a Tangible Game?. 17th IFIP Conference on Human-Computer Interaction (INTERACT), Sep 2019, Paphos, Cyprus. pp.135-155, 10.1007/978-3-030-29384-0_8 . hal-02544614

\section{HAL Id: hal-02544614 \\ https://hal.inria.fr/hal-02544614}

Submitted on 16 Apr 2020

HAL is a multi-disciplinary open access archive for the deposit and dissemination of scientific research documents, whether they are published or not. The documents may come from teaching and research institutions in France or abroad, or from public or private research centers.
L'archive ouverte pluridisciplinaire HAL, est destinée au dépôt et à la diffusion de documents scientifiques de niveau recherche, publiés ou non, émanant des établissements d'enseignement et de recherche français ou étrangers, des laboratoires publics ou privés. 


\title{
How do typically developing children and children with ASD play a tangible game?
}

\author{
Amani Indunil Soysa ${ }^{10000-0001-5252-9510]}$ and Abdullah Al Mahmud ${ }^{\text {[0000-0002-2801-723X] }}$ \\ ${ }^{1}$ Swinburne University of Technology, Australia \\ 1asoysa@swin.edu.au \\ ${ }^{2}$ Centre for Design Innovation (CDI), Swinburne University of Technology, Australia \\ aalmahmud@swin.edu.au
}

\begin{abstract}
A tangible user interface (TUI) brings a strong educational potential for both typically developing children and children with ASD, as it fosters tactile stimulation, development of cognitive skills and perceptual skills. However, no previous research has compared the commonalities and differences of using TUI for both groups in low-resource countries. This study examines the nature of using TUI in children with autism (ages $3-5 ; n=14$ ) compared to typically developing children (ages 3-5; $\mathrm{n}=10$ ) along with their performance in game-play. We used an iPad-based Picture to Object Mapping Activity (POMA) game that utilised tangible components using 30 interactive pretend play toys. We collected video recordings and logs of the POMA application to explore the commonalities and differences of using TUI among both groups of children. Results indicated that children with ASD performed lower than the typically developing children in group activities; however, both groups showed similar performance in individual activities. Furthermore, we observed children with ASD had difficulties in using TUI (e.g. placing the tangibles on the iPad) when compared to typically developing children. Based on our findings, we propose recommendations for developing low-cost TUI for children with ASD in Sri Lanka.
\end{abstract}

Keywords: Tangible user interface (TUI), autism, technology, developing countries, game, tangible interaction

\section{$1 \quad$ Introduction}

Tangible User Interfaces (TUI) have demonstrated its potential in supporting children for their learning activities in the past ten years. Numerous studies have shown that TUI-based tools can be used in educational activities such as geometry training [7], colour matching [31], science activities [42], computer programming [26] for typically developing (TD) children as physical objects interacting with virtual environments can cater playful learning environment [42]. According to Piaget developmental theory, manipulation of concrete physical objects can improve thinking and spatial skills for young children [53]. In addition to such skills, the work on TUI has suggested that TUIs 
might be suitable for engaging children and collaborative learning for children [33]. Therefore, a number of design-focused projects have researched on TUIs to improve social and cognitive skills for young TD children [41] as well as for children with social cognitive impairment such as Autism Spectrum Disorder (ASD) [2, 17, 52]. TUI is particularly beneficial for children with ASD as it supports tactile and sensory stimulation with digital feedback and effects, which are important factors for children with ASD as it can facilitate the sensory needs of children with ASD [30]. Although TUIs are beneficial for both TD children and children with ASD, we are not aware of any study, which has compared the ways of using TUIs among both groups. Therefore, in this paper, we evaluate a TUI-based collaborative educational game called POMA with TD children and children with ASD to explore the commonalities and differences when using TUI by both groups of children.

\subsection{Use of TUI for children with and without ASD}

ASD is a developmental disorder that can appear from a very early stage of childhood and it is associated with cognitive impairment in attention, social communication along with restrictive and repetitive interests and behaviours. Due to such impairments, children with ASD demonstrate problems in social engagement, impatience in turntaking and waiting [10] that could potentially affect their day-to-day activities and their quality of life [25]. Therefore, it is imperative to diagnose children with ASD and direct them to proper intervention programs from a very early stage. These interventions often include tangible toys (i.e. cause and effect toys), visual support (flash cards) to represent both concrete and abstract real-world concepts [4]. For instance, occupational and special education teachers use cognitive therapies like object discrimination with pretend play toys (i.e. plastic toy fruits and vegetables) to empower very young (ages 4-6) children with ASD [55]. Similarly, some special education teachers and general education teachers used Montessori teaching methods and Montessori toys [35], to improve sensor-motor training and spatial abilities via physical objects (toys) that are relatable and natural for children. Further, such Montessori teaching methods highlight the importance of learning through exploration, physical play, repetition and simplicity, not only for TD children but also for children with ASD [5, 24]. For instance, in teaching children to understand the basic geometric shapes, teachers use flashcards containing the picture of the shape to match the correct physical object that represents the geometric shape in the flash card. The strengths of hands-on interaction with physical objects are that they can help understand the representations of relations within and between objects (e.g. shapes) [51]. Such skills are essential for early childhood development for both TD children as well as children with ASD. These significant factors such as learning through physical play, repetition and visual support can be considered when designing technologies and tools targeting young children.

With the advancement of technology, there have been many technological tools designed to support children $[8,28]$. Most of these technological interventions support 2-dimensional learning with visual and auditory support such as touch-based technologies (iPad, tablets and tabletop applications) [36, 54], computer-based technologies (computer software) [21], virtual reality (computer software) [14] etc. 
Such technologies and tools can be beneficial for children especially children with ASD as these tools promote visual learning with immediate feedback. Though these tools and technologies provide interactive visual support for children, these tools lack the benefit of 3D learning and the use of real-world objects that support more effective or more natural learning [46]. TUI-based technologies can effectively be used to avoid such limitations, as it embeds digital technology into graspable forms blending the best of both digital and tangible worlds [31]. Prior research has shown that TUI-based technologies can be used to facilitate a wide array of learning topics from basic puzzles [56] to carpentry [12] for children. These studies focus on improving cognitive skills such as spatial cognition [6] of TD children; however, most research in TUI to support children with ASD mainly involves in skills like social interaction [19, 52] and cognitive skills $[16,45]$. Most of these aforementioned TUI-based tools are designed using passive tangible objects such as toys with RFID-based tokens, magnet sensors and fiducial markers $[23,32]$. These passive tangibles use digital displays such as tabletop devices, computer screens to provide visual and auditory feedback for the children, which enable children to engage in natural and direct interactions [32]. In addition to tabletop-based TUIs, researchers have built smart toys such as Polipo [50], which is an active TUI that offers various multisensory stimuli aimed at promoting finemotor skills of children with ASD. Similarly, researchers have designed active tangible educational interfaces (i.e., "ReduCat") for therapists to engage with children with ASD in educational activities via social stories [2]. Furthermore, Francis et al. [22], has conducted a study with children with and without ASD in the UK to assess the promotion of social behaviour during free play with active TUI (i.e., Topobo) and passive toys (i.e., $\mathrm{LEGO}^{\mathrm{TM}}$ ). This study [22] found out that play with TUI leads to more social play compared to passive toys. Though Francis et al. [22] study provides useful insights on social behaviour during free-play for children with and without ASD, this study does not address the commonalities and differences of using TUI (i.e., how much help is required when using TUI, how TUIs are used among different groups). Further, this study focuses on TUI that is designed for developed countries, which may not be suitable for children in resource-challenged countries.

\subsection{Use of technology for children in low resource countries}

Despite these cited works [16, 19, 45, 50, 52], the field of TUI for children with ASD is still relatively new. Furthermore, most of these studies mentioned earlier mainly focused on children in developing regions like the United States, the United Kingdom using expensive technologies such as tabletop devices. However, there have been a few studies that focused on assistive technology for children with ASD in low resource countries like India including the work conducted by Sharma et al. [43] and Sampath [40] to support decision making, mathematical and social skills of children with ASD. However, all these studies were limited to digital technology, which focused on 2dimensional learning. For countries like Sri Lanka where autism prevalence is 1 in 93 children [38], and we are not aware of any study that focuses on assistive technology for the Sri Lankan children with ASD. Furthermore, there are several challenges of using technology for children with ASD in developing countries like Sri Lanka such as 
limited access to resources, equipment and knowledge may hinder parents and practitioners using technology at home and school setting [1]. For instances, prior studies have found that in Sri Lanka, several vital factors inhibit families from seeking support for their child with ASD including, limited access to services, low-income, lack of knowledge, cultural and religious beliefs and limited services in their native languages [15]. However, Sharma et al. [44] have shown possible guidelines to avoid such challenges. For instance, designing with stakeholders (i.e., therapists and teachers) collaboratively may lead to more technology acceptance within autism communities. Furthermore, designing for diverse learners for collaborative use without individual use (i.e., classroom settings, therapy settings) to facilitate multiple users can also help loweconomic autism communities. Educating parents and practitioners on how to use technology (i.e., limiting technology for 15-30 minutes) to reduce possible harmful effects of using technology for children with ASD (i.e., screen addiction) can also improve the awareness and the use of technology within lower resource regions. Additionally, Sharma et al. highlight that "Giving control to the educators" may also increase the technology acceptance in autism communities [44].

In the face of the challenges mentioned above and lack of research evidence on designing technologies for Sri Lankan children with ASD, we designed a TUI game called POMA (Picture to Object Mapping Activities) [49] to improve social and cognitive skills for Sri Lankan children with ASD. However, we assume that typically developing children in Sri Lanka would be able to use POMA without any difficulties. Therefore, we conducted an exploratory study comparing the use of POMA by TD children and children with ASD to verify our assumption. The primary focus of this study is to explore the commonalities and differences in POMA gameplay between both groups. The findings of the paper and its implications contribute to designing low-cost tangible games to support children in low resource countries.

\section{POMA: Picture to Object Mapping Activity System}

POMA (Picture to Object Mapping Activity) game is a TUI-based educational game running on an iPad that promotes picture to object mapping activities via placing tangible objects on top of the iPad surface. The target group for POMA is Sri Lankan children with ASD in the age of 3-6 years. However, educational and social components of POMA are based on the Sri Lankan standard preschool education system, which is applicable for both TD and children with ASD. Therefore, it is expected that POMA can be played equally by both TD children and children with ASD. The main objective of designing POMA is to get the children engaged in regular learning activities with social interaction in Sri Lankan autism therapy centres. POMA has been designed with consultation of parents and practitioners of Sri Lankan children with ASD. For the complete description of the POMA design process, please to refer our previous work [47-49]. 


\subsection{Features of POMA}

The architecture of POMA has two main parts namely software component, and tangible component (see Fig. 1). The software component is composed of four different activities with six levels. The four activities are related to 1) shapes, 2) fruits, 3) vegetables, and 4) animal identification. During each activity, a tablet application displays a picture related to the activity, and children need to place the appropriate toy on top of the displayed picture. For instance, if the activity is shape identification, a shape related image (i.e., "starfish") will be displayed on the iPad screen. Once the children place the correct-toy (i.e., star-shaped toy) on the iPad screen, children will be reinforced by the iPad and directed to the next shape object. If children were not able to place the correct toy on the iPad screen, iPad would not provide any feedback to the children; however, the same picture will be displayed until children place the correct toy. The game will continue for the predefined duration (default 2 minutes), however, practitioners have the ability to change the game duration for each child. The levels of POMA start from beginner level to more advanced levels from single user modes to multi-user modes (see Fig. 1, 2). When children play the POMA application, practitioners get to select the child preferred background colour, child's level and the activity of their choice. Fig. 1 lists the main activities and levels that are supported by POMA.
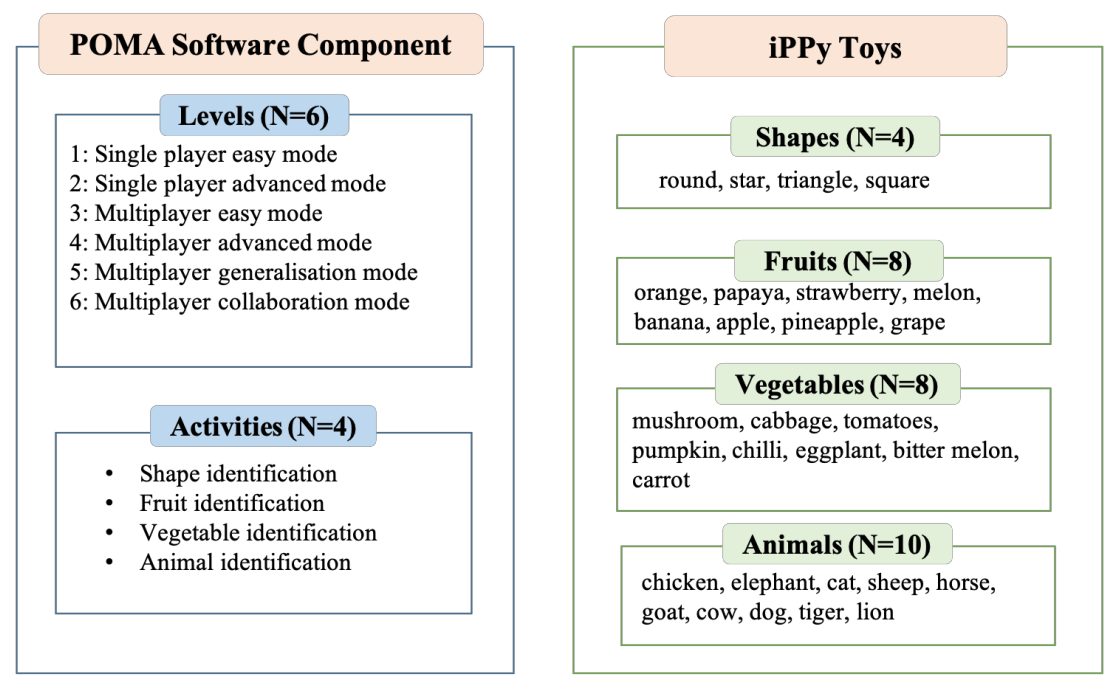

Fig. 1. Architecture of POMA

The tangible component of POMA consists of a set of interactive pretend play toys (iPPy toys) that can communicate with multi-touch surfaces. Tangible components of this prototype are designed using a set of pretend play toys that are commonly used by children with ASD in their therapy sessions. To make these pretend play toys interactive and recognisable by multi-touch surfaces (i.e., iPad) we embedded capacitive touch point patterns on the bottom layer of each interactive toy (see Fig. 2.g, h). To improve 
the safety, robustness and durability of the tangible components as well as to reduce the cost of the tangibles, we have designed iPPy toys to be passive tangibles. Additionally, we also wanted the iPPy toys to be simple and relatable passive objects to avoid any fixation on toys for children with ASD. Passive tangibles are unpowered physical components and are not tightly bound to the digital system [27]. Unlike active tangibles, iPPy toys do not have lights and audio embodied into the physical component. Therefore, the software component of POMA was used to provide audio and visual feedback to the children. To give a variety of iPPy toys for children, in consultation with the Sri Lankan therapists, we selected four different categories of toys that are commonly used by children with ASD in Sri Lanka (see Fig. 1).
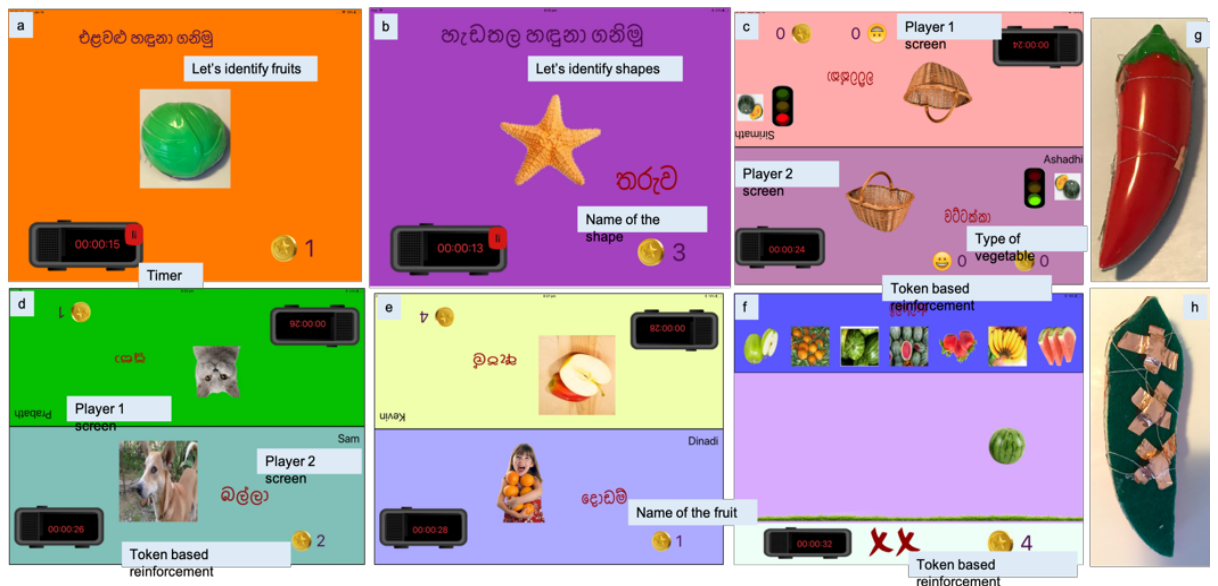

Fig. 2. Levels of POMA and iPPy toys, a) level 1: vegetable identification activities, b) level 2: shape identification activities, c) level 3: vegetable identification basic turn-taking activities, d) level 4: animal identification turn-taking activities, e) level5: fruit identification generalization activities, f) level5: fruits identification collaboration activities; g, h) iPPy chilli-shaped toys.

\section{$3 \quad$ Methodology}

\subsection{Participants}

After obtaining approval from our university ethical review board, we recruited participants from Sri Lankan autism centres and preschools in Colombo. A total of 24 children participated in this study under two groups (group A and B). In group A, 14 children (nine males, five females) from 3-5.5 years $(M=4.8, S D=0.57)$ were recruited from Sumaga autism centre, Kelaniya Sri Lanka. The eligibility requirements for the group A included (a) medical diagnosis of autism according to DSM V diagnosis criteria, (b) diagnosis of autism is mild, (c) verbal communication without the use of an augmentative alternative communication device as verified by the parents, (d) age group of 3-6 years, (e) hearing and vision within normal (6/6 vision, hearing ranging 0 $20 \mathrm{~dB}$ ) limits (aided or unaided) based on parents' report, (f) motor and physical abilities to manipulate a tablet device. In group B ten children (four males and six 
females) from 3-5 years $(M=3.5, S D=0.454)$ were recruited from a Sumaga preschool in Kelaniya Sri Lanka. The eligibility requirement for the group B included (a) no medical diagnosis of any psychological or physiological condition, (b) age group of 3-6 years, (c) hearing and vision within normal (6/6 vision, hearing ranging 0-20 dB) limits (aided or unaided) based on parents' report, and (d) motor and physical abilities to manipulate a tablet device.

In addition to the children, we recruited practitioners (five special education teachers and three primary school teachers) to conduct the study. The recruited practitioners have worked closely with the recruited children and have more than three years of experience working with children.

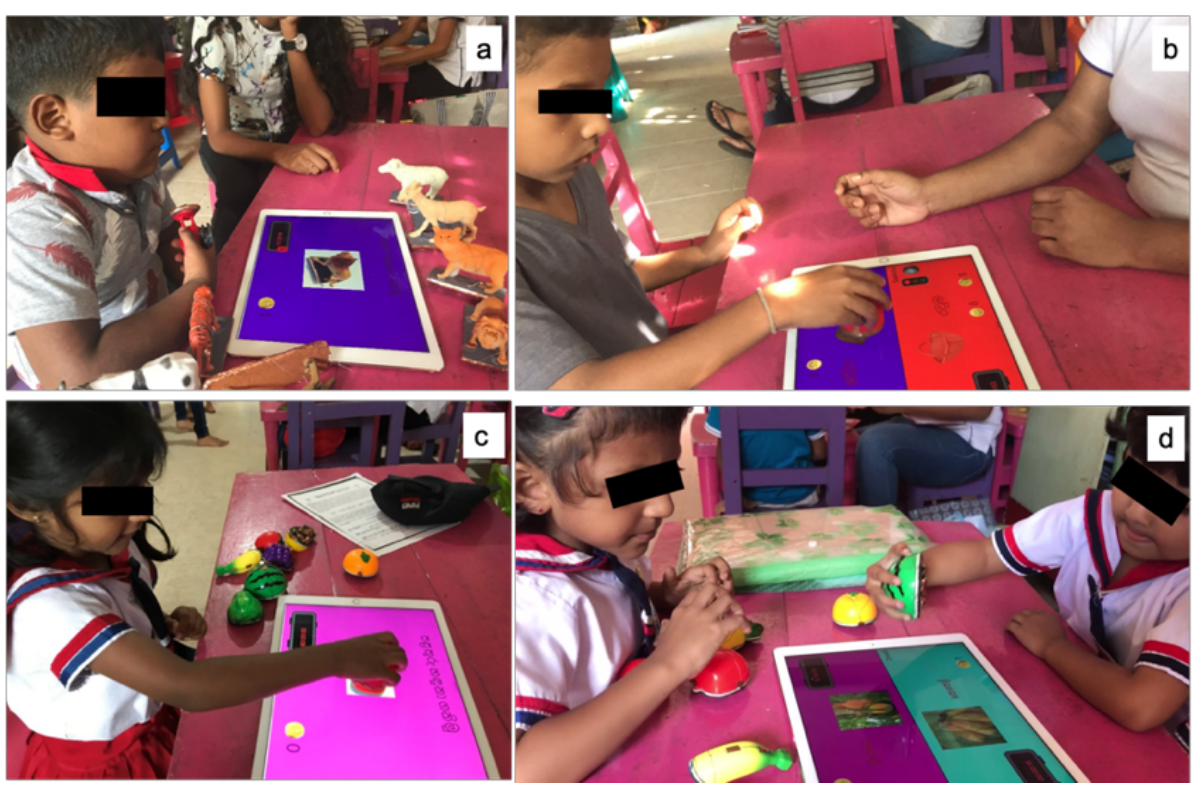

Fig. 3. Children with ASD and typically developing children playing with POMA; a) child with ASD playing POMA-single-player mode, b) child with ASD playing POMA with the practitioner, c) A TD child playing POMA-single-player mode, and d) two TD children playing with POMA.

\subsection{Procedure}

The study was conducted in two different locations in Sri Lanka. For group A, we held the study in the autism centre classroom, in Sumaga autism centre Kelaniya Sri Lanka, and for group B, we held the sessions in the pre-school classroom in Sumaga preschool Kelaniya Sri Lanka. However, both groups of children were in the same age, therefore, both groups followed the similar steps (i.e., single-player to multiplayer modes) during the sessions. At the beginning of the session, the first author described the functionalities of the POMA application and iPPy toys to each practitioner and gave them time to explore POMA installed on an iPad. 
Before conducting the sessions with children, practitioners locked the iPad to the POMA application using the guided access feature of the iPad, which disable the home button of the iPad. This was carried out to prevent children from accessing other apps such as YouTube application during the sessions. Then each practitioner introduced the iPPy toys to each child starting from iPPy shape-toys. Depending on the child's preference practitioners switched the iPPy shape-toys to the other toy categories namely iPPy animal-toys, iPPy vegetable-toys and iPPy fruit-toys. Furthermore, are two main modes of POMA namely single-player mode and multi-player modes. For the singleplayer mode levels (i.e., level 1 and 2), children played with the system by themselves (see Fig. 3.a, c) and practitioners observed and helped the children when needed. For the multiplayer mode levels (level 3, 4, 5, and 6), practitioners selected another child from the same group of the participant to play in the group settings (see Fig 3.d). Furthermore, practitioners played with the children as their partner, whenever a specific child is not able to play with their peers (see Fig 3.b).

Practitioners started playing POMA with children from the single-player mode basic level (Level 1) to more advanced multiplayer modes. If a child performs well at a given level (if the child places the correct objects at least $60 \%$ of the time), he/she will be promoted to the next level by the practitioner. If the child does not perform well, the child will be asked to repeat the level until he/she performs well. Before repeating a level or going to another level, practitioners asked each child if they wanted to continue playing or if they wished to stop playing it. At the end of each activity, children were given 2-7 minutes' break (depending on the child's requirement), before moving to the next activity. After one hour of maximum duration, children were asked to stop playing with POMA by the practitioners to avoid any addictions.

\subsection{Data collection and data analysis}

We collected objective data from the POMA application log and subjective data from the video recordings of all the sessions.

Log data: We collected objective data such as time-taken to complete levels and the total number of levels completed by a child from the POMA system log.

Video recordings: All sessions were video recorded with a total amount of footage of 9 hours and $48 \mathrm{~min}$. Table 1 describes the video coding scheme. We also coded common behaviour patterns of children (i.e., staring at toys, line-up toys) while playing POMA. These behaviour patterns were reported in relation to four main aspects, including; 1) time taken to play the POMA levels, 2) Playing POMA independently, 3) use of the tangible iPPY toys and 4) social engagements via turn-taking and vocal initiation.

We analysed the log data and video recordings against the two groups (TD children and children with ASD). For instance, we calculated the time taken to play POMA and vocal initiations by averaging the time taken to complete each level and averaging the number of vocal initiations for each level. Similarly, we calculated the help required from the practitioners by calculating the average percentage on the number of times practitioners helped children to play POMA from the total number of attempts. For object selection and placement, we calculated the average percentage of correct object placement, the average percentage of correct object selection but incorrect object placement (i.e., upside down placement, finger touching placement), and the average percentage of incorrect object selection from the total number of attempts. Additionally, 
for turn-taking behaviour, we calculated average percentage on the number of times children turn-take the toys willingly with practitioners/peers from the total number of times POMA-game requested to turn-take the toys with the partner. Furthermore, we conducted an inferential statistical analysis using SPSS software where necessary.

Table 1. Video coding scheme

\begin{tabular}{ll}
\hline Codes & Description \\
\hline Help required from the practitioners & $\begin{array}{l}\text { The occurrence of practitioner helping children to } \\
\text { play POMA (i.e., hand holding, pointing) }\end{array}$ \\
& $\begin{array}{l}\text { How children place the iPPy toys on the iPad } \\
\text { screen (i.e., upside down placement, correct } \\
\text { placements and placing toys while touching the } \\
\text { iPad screen) }\end{array}$ \\
Cbject selection and placement & $\begin{array}{l}\text { Child voluntarily giving the toys to their partner } \\
\text { without being prompt } \\
\text { Vocal initiation due to gameplay (i.e., requesting } \\
\text { toys, vocal compliments to the partner; "you can } \\
\text { Vocal initiation }\end{array}$ \\
do this") \\
Common behaviour patterns while playing POMA \\
(i.e., staring at toys, line-up toys).
\end{tabular}

\section{$4 \quad$ Results}

\subsection{Time taken to play the POMA levels}

In general, both typically developing children and children with ASD showed similar capabilities when completing the POMA application. Results indicated that $50 \%$ of the TD children and $57 \%$ of children with ASD were able to complete up to level 5 of POMA application while the remaining children completed only up to level 4 . None of the groups was able to complete level 6 of POMA application within their sessions. Additionally, we also found out that children with ASD spent more time $(M=32.85$ minutes, $S D=7.19)$ while TD children spent lesser time $(M=24.5$ minutes, $S D=4.42)$, even though both groups played similar number of levels (Minimum: 4, Maximum: 5). There was a significant difference on the overall time spent at the $p<0.05$ level for two groups of children $[F(1,22)=10.567, p=0.004]$. 


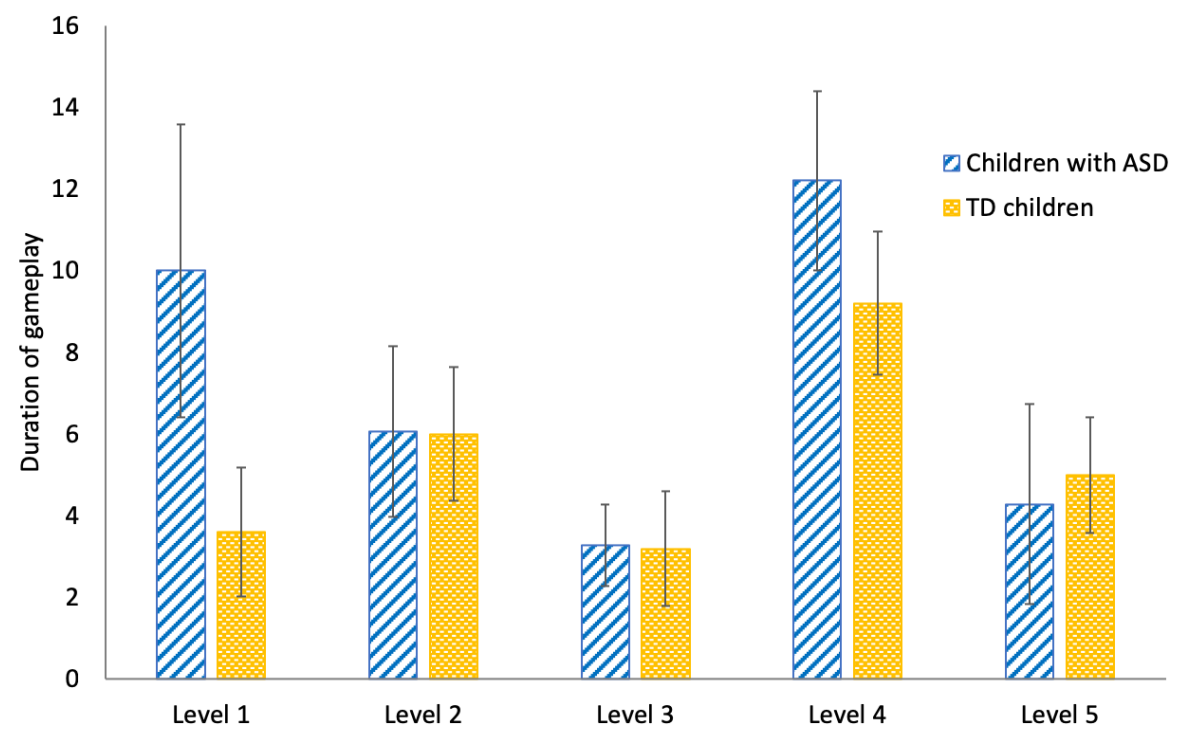

Fig. 4. Average time taken to complete each level of POMA.

We also observed in video recordings that children with ASD spent more time looking and feeling the iPPy toys while playing the initial levels of POMA. For instance, to complete level 1 of POMA children with ASD took considerable time ( $M$ $=10$ minutes; $S D=3.6)$ when compared to TD children $(M=3.6$ minutes, $S D=1.6)$, see Fig. 4. The time taken to complete level 1, therefore, was significant, $F(1,22)=$ 27.6, $p=.000$. However, both groups took the similar amount of time to complete level 2 and 3 and the time taken to complete levels was non-significant (Level 2, $[F(1,22)=$ $0.08, p>0.05]$, Level $3,[F(1,22)=0.31, p>0.05]$. We also observed that both groups took a considerable amount of time (ASD: $M=12.21$ minutes, $S D=2$, TD: $M=9.2$ minutes, $S D=1.75)$ to complete level 4 , which includes turn-taking activities with multiple toys. The time taken to complete level 4 (see Fig. 4 ) was significant, $F(1,22)$ $=12.99, p=0.02$. However, we observed both groups took lesser time (ASD: $M=4.28$ minutes, $S D=2.44$; TD: $M=5$ minutes, $S D=1.4)$ to complete level 5 with no significant difference $p>0.05$.

\subsection{Playing POMA independently}

To explore whether both groups of children can use POMA independently, we calculated the average percentage of help required from practitioners to complete a level. This was calculated by coding the number of times practitioners helped children for each level and a number of time children played without help from the video recordings. Then we calculated the percentage of help required from total attempts for each level. 


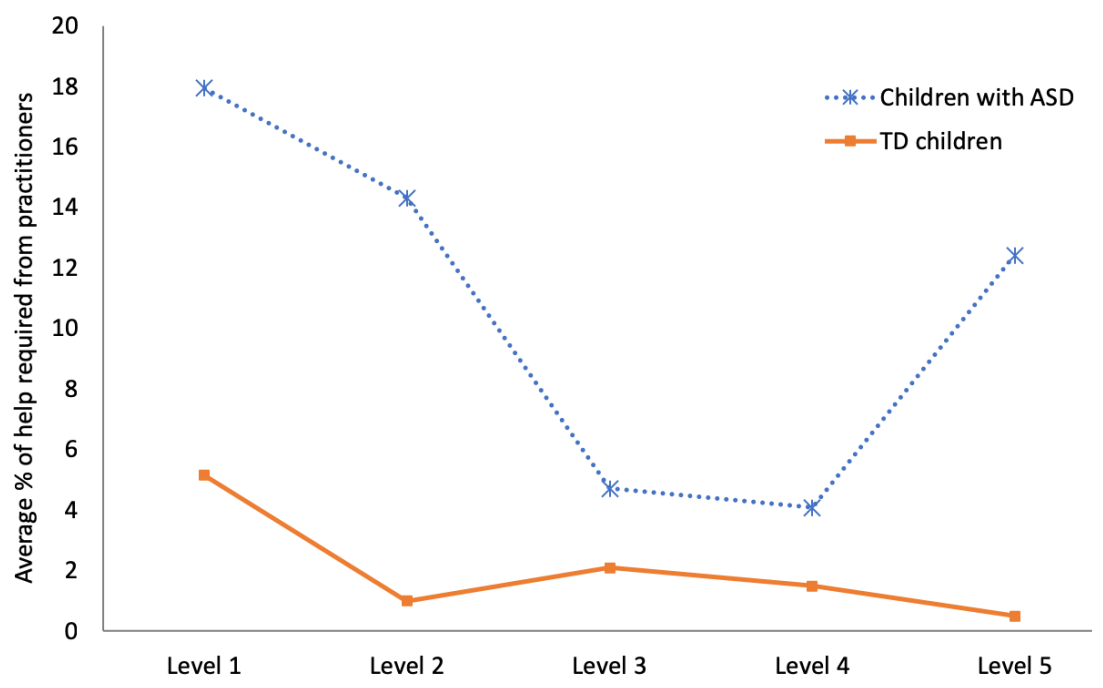

Fig. 5. The average percentage of help required from practitioners for children to play POMA

In general, we found out that both group of children (ASD: $M=17.95 \%, S D=11.5$ and TD children $M=2.5 \%, S D=1.5$ ) required more help from the practitioners to complete the level 1 of POMA (see Fig. 5). However, there was a significant difference between the two groups of children to complete the level one, $\mathrm{F}(1,22)=15.89, p=0.01$. However, after level $1 \mathrm{TD}$ children were much more confident to use POMA independently compared to children with ASD. For instance, to complete level 2, children with ASD required more help $(M=12.1 \%, S D=10.6)$ on average while TD children required lesser help $(M=1 \%, S D=0.94)$. An analysis of variance showed that the help required for children with ASD and TD children to complete level 1 was significant, $[F(1,22)=15.83, p=.001]$. Additionally, we also observed, even though TD children required more help in the initial levels, they required lesser help in more advanced levels. For instance, to play level one TD children required more help ( $M=$ $2.5 ; S D=1.5)$, compared to the help required in level two $(M=2.5 \% ; S D=0.9)$, three $(M=1 \%, S D=0.7)$ and four $(M=2.1 \%, S D=0.8),[F(4,39)=4.31, p=.006]$.

Further, from our video analysis, we found out that children with ASD often (8 times on average per session) got distracted by aligning the toys and looking at the toys. Therefore, practitioners got more involved during play sessions to keep the children with ASD focused on the game.

\subsection{Use of the tangible iPPy toys}

We observed that both groups of children had similar cognitive skills in identifying correct tangible objects of the POMA application. On average 78.3\% $(S D=10.11)$ of the time children ASD and $78.44 \%(S D=4.52)$ of the time TD children were able to identify the correct tangible objects to place it on the iPad screen. Furthermore, we also observed that children with ASD scored less for levels that had multi-user modes (i.e., Level 3, Level 4, Level 5). For instance, on average TD children scored 24.2 points 
$(S D=2.8)$ for Level 3 while on average children with ASD scored $10.466(S D=4.5)$ for Level 3, $[F(1,22)=71.67, p=.000]$.

Additionally, we further observed that $6.38 \%$ of the time children with ASD were able to identify the correct toy, but not able to place it correctly on the iPad screen. From the video data coding, we identified that children with ASD were unable to place the toys due to two main reasons. 1) FT- Fingers touch the iPad screen when placing the iPad; therefore, the touch point pattern gets distorted. 2) UD- putting the toys upside down; therefore, the iPad cannot recognise the touch point pattern. From the incorrect object placement, we found out that most of the FT-placements $(M=28.9 \%, S D=10.4)$ were for vegetable type-iPPy toys and most $(M=16.7 \%, S D=20.1)$ of the UD placements were for shape-type iPPy toys. When compared to children with ASD, TD children had very low $(M=0.105 \%, S D=0.12)$ incorrect object placement $[F(1,22)$ $=14.04, p=.001]$. Further, we observed from our video recordings that once typically developing children learn to place the tangible toys correctly in Level 1, and they avoided FT and UD placement completely.

In addition to FT and UD placements, we also observed that children with ASD spent some time ( $M=87.64$ seconds, $S D=62.4)$ aligning the toys properly, keeping the toys in a straight line after placing them on the iPad. Furthermore, we observed that some children with ASD $(n=7)$ got frustrated when they cannot place animal toys in the standup position throughout the levels. However, we did not find such behaviour in TD children.

\subsection{Social engagement via turn taking and vocal initiation}

From the third level, POMA embeds social components using multiplayer functionalities along with turn-taking the tangible objects. From the practitioner observations, we found out that only two children with ASD were able to play multiplayer activities among each other (with peers) and the remaining children with ASD played the multi-player activities with their respective practitioners. In contrast to children with ASD, all TD children were able to play multiplayer activities of POMA with their peers. To evaluate the social engagement throughout the sessions, we coded the two variables such as 1) the number of times children turn-take the toys willingly with practitioners/peers and cooperatively played with others and 2) the number of social interactions made via vocal initiation during the playtime.

The average percentage of turn-taking was determined by calculating the percentage of toys shared willingly by the children with their partner (i.e., peers or practitioner) with the total number of times POMA-game requested to turn-take the toys with the partner. In general, both children with ASD and TD children were able to share the physical toys among each other. We also observed (see Fig. 6) that TD children were more willingly turn-taking $(M=98.2 \%, S D=1.92)$ compared to children with ASD ( $M$ $=81.5 \%, S D=19.02)$. However, we did not observe any significant differences between both groups of children $[F(1,22)=7.643, p=.006]$. Additionally, we observed that over time both groups of children improved their willingness to take-turns among each other via sharing their toys. Furthermore, we noticed that all children who played level five (TD: $n=5$; ASD: $n=5$ ) took turns willingly without any hesitation. 


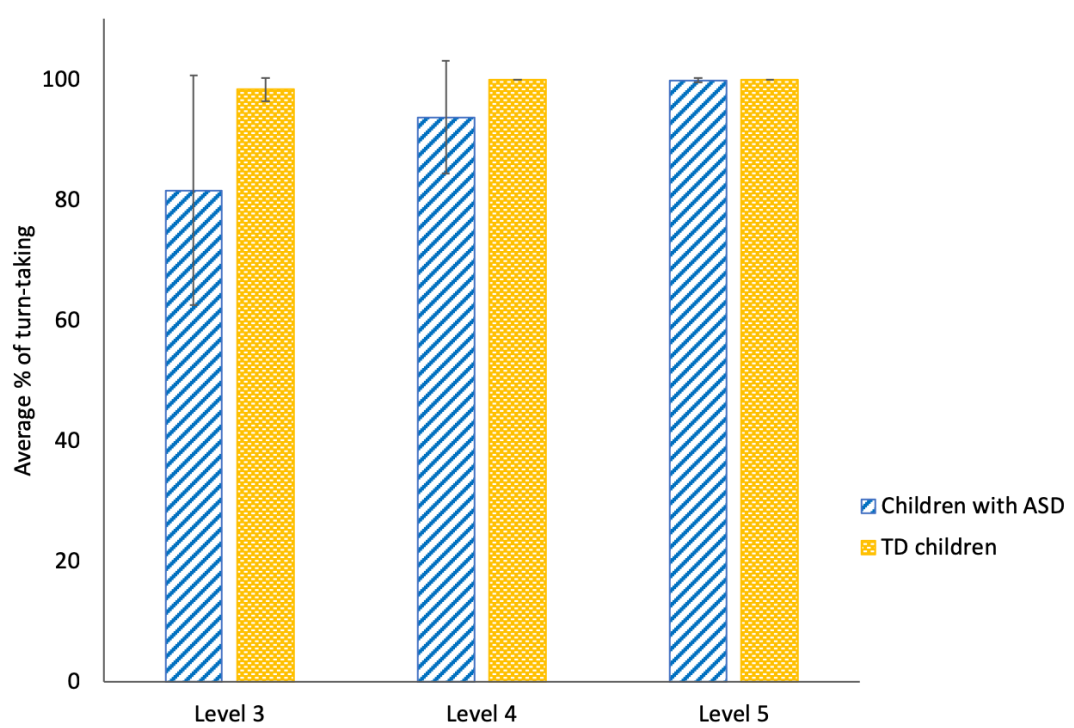

Fig. 6. Average percentage (\%) of turn-taking during different levels

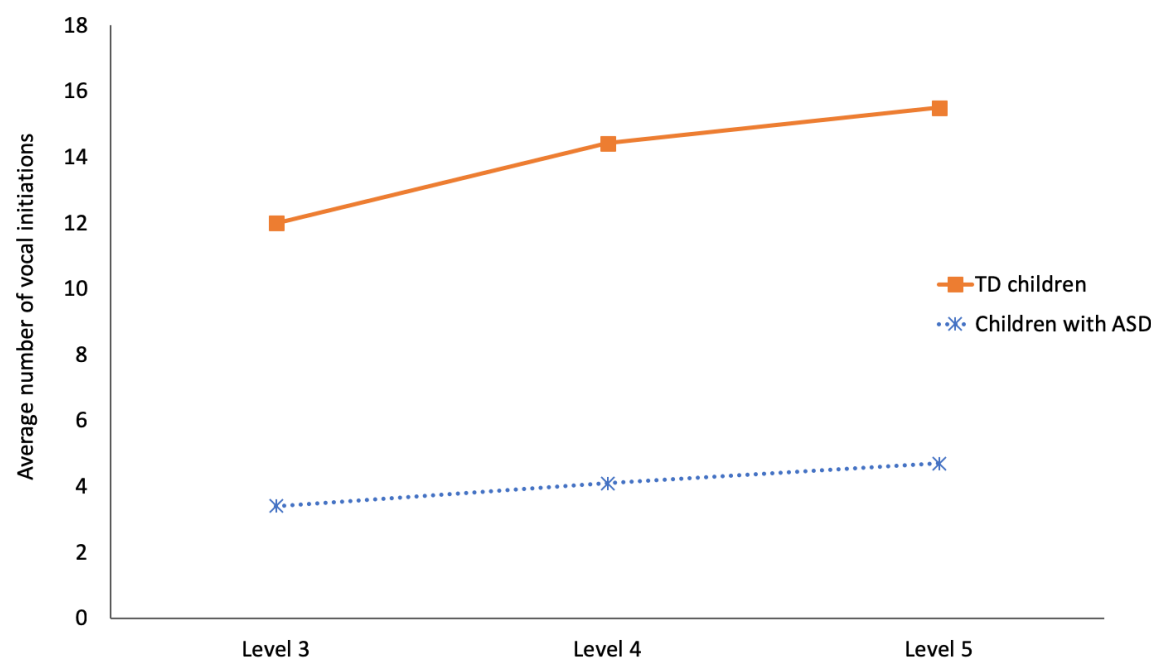

Fig. 7. Average number of vocal initiations per level

Even though children with ASD were willingly sharing the toys among each other, we observed very few vocal initiations from children with ASD when compared to typically developing children (See Fig. 7). We observed significant differences in vocal initiation between the two groups. For instance, for the level 1, children with ASD spoke around 3-4 times on average $(S D=2.4)$ while TD children-initiated 
conversations around 8-9 times per session $[F(1,22)=26.8, p=.000]$, indicating TD children are more verbal during POMA gameplay compared to children with ASD. Though children with ASD had limited verbal communication between their partner, we also observed they kept on requesting for the toys using hand gestures. We also observed that both groups increased their verbal initiations throughout the levels (see Fig. 7). Additionally, we found that TD children helped each other by complimenting each other ("good job"), repeating the reinforcements generated by the iPad and also saying what objects needs to be picked verbally. In contrast to TD children, children with ASD showed less interesting in helping each other verbally; however, we observed that they kept on repeating the audio feedback/reinforcements given by the iPad when his/her partner is doing well (i.e., "Well done", "You got it"). Table 2 summarizes the study findings by highlighting the commonalities and differences in gameplay between the two groups.

Table 2. Commonalities and differences in POMA-gameplay between TD children and children with ASD in Sri Lanka

Commonalities

Both TD children and children with ASD were able to complete only unto level 5 (50\% of the TD children and $57 \%$ of the children with ASD completed level 5 the others completed up to level 4).

Both TD children and children with ASD had the similar level of cognitive skills when

2 identifying the iPPy toys correctly $(78.545 \%$ of the time TD children and $79.23 \%$ of the time children with ASD identified the objects correctly).

3 Both TD children and children with ASD were able to share the objects among the other players and turn-take when playing multiplayer modes.

Differences

Children with ASD took a significant amount to complete level 1 and 4 when compared

1 to TD children.

Even though both groups had similar cognitive functionalities, children with ASD had

2 difficulties placing the objects on the iPad screen ( $6.38 \%$ of the time).

Children with ASD required more help from the practitioners compared to TD children (children with ASD requested help 11.6\% of the time throughout all the levels while TD children only needed help $1.2 \%$ of the time in the initial levels).

TD children had sufficient amount of social engagement via vocal initiation (8-10 times

4 per session on average. However, children with ASD had a limited amount of social engagement via vocal initiation (4-5 times per session on average).

5 Only two children with ASD were able to play multiplayer modes with their peers. However, all TD children were able to play multiplayer modes with their peers. 


\section{Discussion}

In the following section, we reflect on the key findings and provide implications for designing TUI for children in Sri Lanka.

\subsection{Reflection on the key findings}

In this paper, we have presented how TD children and children with ASD in Sri Lanka play a TUI game, and we have illustrated the commonalities and differences in gameplay for both group of children. This study provides further evidence that well-designed TUI-based educational systems can leverage playful learning among children and promote social interaction in children. By comparing the progress reports of children with ASD and TD children, we were able to observe that cognitive skills (i.e., shape identification) overlapped between the two groups. Children in both groups were able to identify shapes, animals, veggies and fruits around $78-79 \%$ of the time. Hence, this study provides further support for the use of TUI in education for TD children [41] and children with ASD [45]. However, we observed qualitative differences between the two groups in the way they place the tangible toys on the iPad screen. For instances, $6.83 \%$ of the time children with ASD were not able to place the tangible toys even after correctly identifying them. We observed two reasons for such behaviour, 1) placing the objects while the fingertips were touching the iPad screen, and 2) putting the objects upside down. For both types of placements, iPad cannot identify the tangible toys as the touch point pattern gets distorted. In addition to incorrect toy placement on the iPad, we also observed children with ASD tend to line-up the tangibles during play time. The reason for behaviours like upside-down (UT) placement and line-up toys can be due to visual stimming that can be seen in many children with ASD due to sensory processing disorders [9]. Sensory processing issues occur in $92 \%$ of the individuals with ASD [34] leaving spectrum of strengths and deficits such as visual stimming, fine-motor movements $[29,39]$ and tactile profile. We believe that our recruited children with ASD had sensory processing issues that caused problems when placing slender surface toys such as fruits and vegetables on the iPad screen. In addition to object placement, we also observed that children with ASD required more time to complete the beginner levels of POMA when compared to TD children. The reasons for this may be due to their fine-motor skill limitations [29, 39], the unfamiliarity of the game and sensory integration deficits [29]. However, it is worth noticing that despite their sensory issues, children with ASD were able to complete the later levels (level 5) slightly faster than the TD children. The reason for this might be due to the predictability and the familiarity gained by playing the initial levels that led children with ASD to focus and concentrate more on the game [20].

Concerning independent playing, we observed both groups required help from practitioners when playing level 1 of POMA (children with ASD: 17.95\% of the time and TD children $5.15 \%$ of the time). The main reasons for this might be because of the unfamiliarity of the POMA game since both groups of children were using TUI for the first time. Another reason for such behaviour might be because the majority of the Sri Lankan learning environments are teacher-centric and children in Sri Lanka rely on 
their teachers when learning new concepts [13]. We also found that over the time children required less help from the practitioners especially the TD children. TD children were found collaborating and turn-taking with each other without being forced by their partner to share their toys with their partner. However, we also observed even after level 1, children with ASD required additional help from the practitioners. For instance, we found children with ASD sometimes tend to get distracted by lining-up toys and staring at the toys, hence, practitioners were required to bring back the focus to the game. This may be one of the reasons why children with ASD took more help in level 5.

Similar to a prior study [18], we found that children with ASD show some degree of social interaction through turn-taking and co-operative play during multiplayer mode sessions of POMA. We also consider that enforcing co-operative play via multiplayer modes can leverage social interactions. For instance, our multiplayer mode play sequence findings suggest that level 3,4 and 5 offer more opportunities for turn-taking and sharing in TD children as well as children with ASD. However, we also observed that limited vocal initiation in children with ASD when compared to TD children during multiplayer modes of POMA. Such behaviour is not surprising for children with ASD since children with ASD have limited verbal skills compared to TD children [3]. Despite having limited verbal abilities, children with ASD were able to socially engage among their partners using hand gestures while improving their verbal skills throughout the levels. Another important finding from this study is that only two children with ASD were able to play with their peers without adult directions, and remaining children with ASD $(\mathrm{n}=12)$ required practitioners as their partners when playing the multiplayer modes. In contrast to our findings, prior studies have shown that adult directed play goal is not always necessary to promote socially-oriented free play when playing with construction-based TUI, especially when there are no specific goals [22]. However, in our study, we found out practitioner involvement was necessary to guide them while playing and keeping their focus on educational activities. The reasons for children with ASD required practitioner as their partner instead of their peers might be related to the characteristics of ASD such as the need for familiarity, predictability and consistency [11] that can be expected from their practitioners instead of their peers.

\subsection{Design implications for developing TUI for children with ASD}

Based on our findings, we have listed a set of guidelines and lessons learned as implications for designing passive TUI for children. Some of these guidelines are specific to children with ASD, while others may apply to all children.

Socialisation: Percentage of social interactions via turn-taking and sharing was much higher than we envisioned during the design process of POMA. We observed both groups of children had above $80 \%$ turn-taking and sharing during the initial levels and improving this skill over-time up to $99 \%$. The main reason for such success can be due to our digital interface, where we enforce parallel play with turn-taking through twoplayer functionalities. Additionally, we have also divided the POMA interface into two parts assigning collaborative roles to each player, which locks/unlocks the touch surface upon sharing and turn-taking. We also provided one set of toys at a given time to add 
the element of scarce resources. Hence, children had to collaborate to succeed in the game. Due to the limited resources (iPPy toys) and token-based reinforcement points, it is known to trigger competitive behaviour in children [37]. However, as seen in prior TUI-based autism studies [22, 50], our study provides further support that TUI-based systems promote collaborative elements in children instead of competition not only for children with ASD but also for TD children.

Structure of the tangibles: The structure of the tangibles was an essential factor that significantly enhanced the progress of the child when using POMA. The central technology behind our passive tangible toys (iPPy toys) is touch point pattern matching. The objects on the digital screen are identified by matching the touch point pattern embedded under each toy. Further, we designed the iPPy toys rounded, graspable and tactile stimulating to both groups of children, since most children with ASD has sensory integration issues and limited fine-motor skills [29, 39]. However, we observed two issues when children with ASD were placing the iPPy toys on the digital display. The first issue we saw was, some of the times children with ASD placed the shape-related toys upside down on the digital screen. Hence, the touch point pattern could not be recognised by the system. To avoid such issues, we propose to provide more cues in the POMA applications in Graphics Interchange Format (GIF) demonstrating how to keep the toys properly on the iPad screen during the initial levels of POMA. The second issue we saw was, children with ASD tend to place their fingertips on the digital surface when placing the slender surface toys (fruits and vegetables) on the iPad that in turn, would modify the touch point pattern for identification. To avoid such issues, we propose to add an additional layer using non-conductive materials (i.e., plastic) around slender surface toys. This layer would prevent fingertips from being touched when placing the toys on the iPad screen.

Sustaining attention: Even though the iPPy toys are passive tangibles (without lights and audio), children were distracted by the iPPy toys and paid more attention to the structure of the iPPy toys and keeping the iPPy toys line-up or stand-up position regardless of placing the toys on the digital surface. One reason for this could be that digital interface of POMA keep on waiting for children to keep the toys on the screen without trying to get the child's attention when in idle. It might be more appropriate to provide audio-visual feedback to the children when the iPad is idle to get the attention back to the game.

\subsection{Strengths and limitations}

One limitation of this study is the unequal sample size with fewer TD children and more children with ASD. Although replication with the same number of children in both groups is required, this study offers preliminary evidence that both groups of children can play POMA effectively and POMA can facilitate social interaction and cognitive skills of children with ASD and TD children. In our study, for the ASD group, we had more boys $(n=9)$ compared to girls $(n=5)$. This may have an impact on the generalizability of our results. During the study period due to the unavailability of female participants at the therapy centres, we were not able to recruit more female participants. Furthermore, we also observed that $85.7 \%$ of the children played multi- 
player activities with their teachers while all TD children played multi-player activities with their peers, which may have some effect on their social engagement. However, due to the social impairments of children with ASD and the limited time given to play with POMA, most children with ASD were unable to play POMA (multi-player modes) with their peers. Therefore, practitioners took the role of being the partners of children with ASD who could not play with each other.

\section{Conclusion and Future work}

In this paper, we have presented how children with ASD and TD children play a lowcost TUI-based game called POMA. Our results show that both groups of children were able to complete up to level 5 of POMA successfully and had similar success in object identification. However, children with ASD took a significant amount in completing levels compared to TD children. Furthermore, children with ASD showed difficulties in keeping the tangible toys on the iPad screen compared to TD children. Additionally, we found that children with ASD needed more help from the practitioners to complete the POMA levels and had lesser social interactions compared to TD children. However, we also observed children with ASD needed less help, less time to complete POMA levels and improve social interactions throughout the levels. Based on our findings, we have provided a set of guidelines for designing TUIs for children with ASD in lowresource countries. In our future work, we hope to improve POMA according to the findings of the current study and evaluate POMA in therapeutic settings for a more extended period to understand the effects of POMA in daily autism therapy practices.

\section{Acknowledgement}

We would like to acknowledge the support extended by the founder Mrs. Swarna Jayawardene and her staff of Sumaga Autism Centre and Sumaga pre-school, Kelaniya, Sri Lanka for this study. We are also grateful to the practitioners, parents and their children who took part in this study.

\section{References}

1. Ahmad, F.K., Use of assistive technology in inclusive education: Making room for diverse learning needs. Transcience, 2015. 6(2): p. 62-77.

2. Alessandrini, A., et al. Designing ReduCat: Audio-Augmented Paper Drawings Tangible Interface in Educational Intervention for High-Functioning Autistic Children. in Proceedings of The 15th International Conference on Interaction Design and Children. 2016. ACM.

3. Anderson, D.K., et al., Patterns of growth in verbal abilities among children with autism spectrum disorder. Journal of consulting and clinical psychology, 2007. 75(4): p. 594. 
4. Asher, A., Book Review: Visual supports for people with autism: A guide for parents and professionals. The Canadian Journal of Occupational Therapy, 2017. 84(3): p. 148.

5. Bartak, L. and M. Rutter, Special educational treatment of autistic children: A comparative study-1. Design of study and characteristics of units. Journal of Child Psychology and Psychiatry, 1973. 14(3): p. 161-179.

6. Baykal, G.E., et al., A review on complementary natures of tangible user interfaces (TUIs) and early spatial learning. International journal of child-computer interaction, 2018.

7. Bonnard, Q., et al. Tangible paper interfaces: interpreting pupils' manipulations. in Proceedings of the 2012 ACM international conference on Interactive tabletops and surfaces. 2012. ACM.

8. Boucenna, S., et al., Interactive technologies for autistic children: A review. Cognitive Computation, 2014. 6(4): p. 722-740.

9. Cardinaux, A. and T. Gandhi, Reduced Anticipatory Responses during Dynamic Object Interactions in Autism.

10. CDC. Autism Spectrum Disorders: Data and Statistics. 2016 July 11, 2016 [cited 2016; Available from: http://www.cdc.gov/ncbddd/autism/data.html.

11. Church, C., S. Alisanski, and S. Amanullah, The social, behavioral, and academic experiences of children with Asperger syndrome. Focus on autism and other developmental disabilities, 2000. 15(1): p. 12-20.

12. Cuendet, S., et al., An integrated way of using a tangible user interface in a classroom. International Journal of Computer-Supported Collaborative Learning, 2015. 10(2): p. 183-208.

13. Dahanayake, S., Implementation of the Philosophical Concept of Student Centred Education at Senior Secondary Level. 2006.

14. Didehbani, N., et al., Virtual reality social cognition training for children with high functioning autism. Computers in Human Behavior, 2016. 62: p. 703-711.

15. Eng, B. and K.E. Foster, Assessing the Accessibility and Integration of Community Resources for Autism in Sri Lanka. 2018.

16. Escobedo, L., et al., Smart objects to support the discrimination training of children with autism. Personal and ubiquitous computing, 2014. 18(6): p. 1485-1497.

17. Farr, W., N. Yuill, and H. Raffle, Social benefits of a tangible user interface for children with autistic spectrum conditions. Autism, 2010. 14(3): p. 237-252.

18. Farr, W., N. Yuill, and H. Raffle, Social benefits of a tangible user interface for children with autistic spectrum conditions. Autism, 2010.

19. Farr, W.J.2011. Tangible user interfaces and social interaction in children with autism. University of Sussex.

20. Ferrara, C. and S.D. Hill, The responsiveness of autistic children to the predictability of social and nonsocial toys. Journal of autism and developmental disorders, 1980. 10(1): p. 51-57.

21. Fletcher-Watson, A targeted review of computer-assisted learning for people with autism spectrum disorder: Towards a consistent methodology. Review Journal of Autism and Developmental Disorders, 2014. 1(2): p. 87-100. 
22. Francis, G.A., et al., Do Tangible User Interfaces promote social behaviour during free play? A comparison of autistic and typically-developing children playing with passive and digital construction toys. 2018.

23. Gelsomini, M. Reflex: Learning Beyond the Screen in a Simple, Fun, and Affordable Way. in Extended Abstracts of the 2018 CHI Conference on Human Factors in Computing Systems. 2018. ACM.

24. Gustafsson, C., Montessori Education, in International Handbook of Early Childhood Education. 2018, Springer. p. 1439-1456.

25. Heyvaert, M., et al., Efficacy of behavioral interventions for reducing problem behavior in persons with autism: An updated quantitative synthesis of single-subject research. Research in Developmental Disabilities, 2014. 35(10): p. 2463-2476.

26. Horn, M.S., R.J. Crouser, and M.U. Bers, Tangible interaction and learning: the case for a hybrid approach. Personal and Ubiquitous Computing, 2012. 16(4): p. 379-389.

27. Horn, M.S., E.T. Solovey, and R.J. Jacob. Tangible programming and informal science learning: making TUIs work for museums. in Proceedings of the 7th international conference on Interaction design and children. 2008. ACM.

28. Hutchby, I. and J. Moran-Ellis, Children, technology and culture: The impacts of technologies in children's everyday lives. 2013: Routledge.

29. Jasmin, E., et al., Sensori-motor and daily living skills of preschool children with autism spectrum disorders. Journal of autism and developmental disorders, 2009. 39(2): p. 231-241.

30. Joosten, A.V. and A.C. Bundy, Sensory processing and stereotypical and repetitive behaviour in children with autism and intellectual disability. Australian occupational therapy journal, 2010. 57(6): p. 366-372.

31. Kubicki, S., et al., RFID interactive tabletop application with tangible objects: exploratory study to observe young children' behaviors. Personal and Ubiquitous Computing, 2015. 19(8): p. 1259-1274.

32. Marco, J., E. Cerezo, and S. Baldassarri, Bringing tabletop technology to all: evaluating a tangible farm game with kindergarten and special needs children. Personal and ubiquitous computing, 2013. 17(8): p. 1577-1591.

33. Marshall, P. Do tangible interfaces enhance learning? in Proceedings of the 1st international conference on Tangible and embedded interaction. 2007. ACM.

34. Mayes, S.D., Brief Report: Checklist for Autism Spectrum Disorder: Most Discriminating Items for Diagnosing Autism. Journal of autism and developmental disorders, 2018. 48(3): p. 935-939.

35. Montessori, M., The montessori method. 2013: Transaction publishers.

36. Muharib, R., et al., Effects of Functional Communication Training Using GoTalk NowTM iPad® Application on Challenging Behavior of Children With Autism Spectrum Disorder. Journal of Special Education Technology, 2018: p. 0162643418783479.

37. Pappert, A.T., A. Williams, and C. Moore, The influence of competition on resource allocation in preschool children. Social Development, 2017. 26(2): p. 367-381.

38. Perera, H., K. Wijewardena, and R. Aluthwelage, Screening of 18-24-month-old children for autism in a semi-urban community in Sri Lanka. Journal of tropical pediatrics, 2009: p. fmp031. 
39. Provost, B., B.R. Lopez, and S. Heimerl, A comparison of motor delays in young children: autism spectrum disorder, developmental delay, and developmental concerns. Journal of autism and developmental disorders, 2007. 37(2): p. 321-328.

40. Sampath, H., J. Sivaswamy, and B. Indurkhya, Assistive systems for children with dyslexia and autism. ACM Sigaccess Accessibility and Computing, 2010(96): p. 3236.

41. Schneider, B., et al., Benefits of a tangible interface for collaborative learning and interaction. IEEE Transactions on Learning Technologies, 2011. 4(3): p. 222-232.

42. Shaer, O., et al., Designing reality-based interfaces for experiential bio-design. Personal and Ubiquitous Computing, 2014. 18(6): p. 1515-1532.

43. Sharma, S., et al. Gesture-based interaction for individuals with developmental disabilities in India. in Proceedings of the 18th International ACM SIGACCESS Conference on Computers and Accessibility. 2016. ACM.

44. Sharma, S., et al., Designing Gesture-Based Applications for Individuals with Developmental Disabilities: Guidelines from User Studies in India. ACM Transactions on Accessible Computing (TACCESS), 2018. 11(1): p. 3.

45. Sitdhisanguan, K., et al., Using tangible user interfaces in computer-based training systems for low-functioning autistic children. Personal and Ubiquitous Computing, 2012. 16(2): p. 143-155.

46. Sluis, R., et al. Read-It: five-to-seven-year-old children learn to read in a tabletop environment. in Proceedings of the 2004 conference on Interaction design and children: building a community. 2004. ACM.

47. Soysa, A.I. and A. Al Mahmud. Beyond Digital Displays: Design Considerations for Tablet Applications Targeting Children with ASD in Sri Lanka. in Extended Abstracts of the 2018 CHI Conference on Human Factors in Computing Systems. 2018. ACM.

48. Soysa, A.I. and A.A. Mahmud. Assessing tablet applications focused on social interactions: what functionalities do Sri Lankan practitioners want for children with ASD? in Proceedings of the 30th Australian Conference on Computer-Human Interaction. 2018. ACM.

49. Soysa, A.I., A.A. Mahmud, and B. Kuys. Co-designing tablet computer applications with Sri Lankan practitioners to support children with ASD. in Proceedings of the 17th ACM Conference on Interaction Design and Children. 2018. ACM.

50. Tam, V., M. Gelsomini, and F. Garzotto. Polipo: a Tangible Toy for Children with Neurodevelopmental Disorders. in Proceedings of the Tenth International Conference on Tangible, Embedded, and Embodied Interaction. 2017. ACM.

51. Verdine, B.N., et al., Finding the missing piece: Blocks, puzzles, and shapes fuel school readiness. Trends in Neuroscience and Education, 2014. 3(1): p. 7-13.

52. Villafuerte, L., M. Markova, and S. Jorda. Acquisition of social abilities through musical tangible user interface: children with autism spectrum condition and the reactable. in CHI'12 Extended Abstracts on Human Factors in Computing Systems. 2012. ACM.

53. Wadsworth, B.J., Piaget's theory of cognitive and affective development: Foundations of constructivism. 1996: Longman Publishing. 
54. Whitehouse, A.J., et al., A randomised controlled trial of an iPad-based application to complement early behavioural intervention in Autism Spectrum Disorder. Journal of Child Psychology and Psychiatry, 2017.

55. Wimpory, D.C., R.P. Hobson, and S. Nash, What facilitates social engagement in preschool children with autism? Journal of Autism and Developmental Disorders, 2007. 37(3): p. 564-573.

56. Xie, L., A.N. Antle, and N. Motamedi. Are tangibles more fun?: comparing children's enjoyment and engagement using physical, graphical and tangible user interfaces. in Proceedings of the 2nd international conference on Tangible and embedded interaction. 2008. ACM. 